\title{
Design of Robust Test Criteria in Analog Testing
}

\author{
Walter M. Lindermeir \\ Department of Electrical Engineering, Institute of Electronic Design Automation \\ Technical University of Munich, 80290 Munich, Germany
}

\begin{abstract}
Test design of analog circuits based on statistical methods for decision making is a topic of growing interest. The major problem of such statistical approaches with respect to industrial applicability concerns the confidence with which the determined test criteria can be applied in production testing. This mainly refers to the consideration of measurement noise, to the selected measurements, as well as to the required training and validation samples. These crucial topics are addressed in this paper. On exploiting experience from the statistical design of analog circuits and from pattern recognition methods, efficient solutions to these problems are provided. A very robust test design is achieved by systematically considering measurement noise, by selecting most significant measurements, and by using most meaningful samples. Moreover, parametric as well as catastrophic faults are covered on application of digital testing methods.
\end{abstract}

\section{Introduction}

With growing complexity and shrinking device dimensions, circuit performances are becoming increasingly sensitive to inherent fabrication deviations. Consequently, parametric faults are becoming more and more important. This leads to two different strategies in the test design for analog circuits.

On the one hand, it is postulated that the effects of parametric faults are completely eliminated by statistical design for manufacturability [1]. Thus, only catastrophic faults need to be considered in testing [2].

On the other hand, it is claimed that for many designs it is neither desirable nor feasible to eliminate all parametric faults by statistical design [3]. In this situation, one is faced with the problem of designing tests for parametric faults.

In [3], it is stated that increasing the performance of the test procedure is often more economical than avoiding parametric yield loss. So, the need for efficient test methods with respect to parametric faults arises. The detection of parametric faults is regarded as a much more difficult task than the detection of catastrophic faults [4].
Commonly, parametric testing of analog circuits is done by verifying all circuit specifications. This is called specification testing [4] or functional testing [5]. Here, a major concern is the minimization of production testing time. In [4], an optimal ordering of functional tests is determined. In [5], an approach called 'Predictive Subset Testing' is presented: a subset of functional tests is determined that is sufficient for testing the circuit. These approaches are efficient in reducing testing time, when at least a subset of specified performances can be measured.

In general, specification testing suffers from two major drawbacks: First, the testing environment usually differs from the operational environment. The specifications of a circuit refer to the operational environment, e.g. a given load. In specification testing, the operational environment needs to be reproduced on the test equipment. This is difficult, if e.g. the input characteristics of the test equipment differs from the specified load. Thus, specification testing generally requires specialized and expensive test equipment. Second, one of the main design goals is to make the specified performances insensitive and robust to fluctuations of the manufacturing process [6]. This is disadvantageous in testing, as measurement errors can mask the effects of parametric faults. So, in order to cut the high costs of analog testing, it seems reasonable to search for alternatives to specification testing.

In this situation, statistical methods for decision making are often applied to derive test criteria for the detection of parametric faults based on preferred measurements $[7,8,9]$. In order to obtain an applicable test design, the performance of the determined test criteria has to be investigated, not only for the nominal case but also in the presence of measurement noise. Moreover, as actually contracted specifications need to be guaranteed to the customer, a test design based on a fault model strongly correlated to specification-based test procedures [2] needs to be performed. But if test criteria are designed to correlate to given specifications, the robustness of the test design becomes an especially crucial point.

This paper presents a new and very efficient approach 
to the design of robust test criteria for analog testing. Inevitable measurement errors are systematically considered in the test design stage and their effect on the quality of the test criteria is investigated. Based on the concept of feature selection [10], a novel and very efficient heuristic for problem-specific measurement selection is developed. On application of this heuristic, the trade off between the number of required measurements, i.e. test cost, and test quality can be interactively controlled. The less measurements are considered, the higher is the estimation accuracy, i.e. the robustness, in the design of statistical decision criteria. Moreover, a novel sampling strategy is presented for the generation of training and validation data sets that provides especially meaningful information for the design of the statistical decision criteria. This sampling strategy is both very efficient and directly physically interpretable in terms of process drift. By this means, the resulting test design can be shown to be very robust with respect to measurement errors. On solving a fault covering problem for catastrophic faults, a very high number of detected catastrophic faults is achieved at low additional test cost. On integrating the proposed approach into the test design stage, specification testing could possibly be avoided and test cost could be significantly reduced.

\section{Starting point and basic relationships}

In this section, the basic relationships for the design of test criteria will be introduced. The presented approach is based on a parametric fault model that builds up a relation between the specified performances $\boldsymbol{p}$ of the circuit, the statistical parameters $\boldsymbol{x}$ that represent fluctuations of the manufacturing process, and the $n_{m}$ measurements $\boldsymbol{m}$ of the circuit $[7,11]$. In each of these three domains, a region of fault free circuits is defined as the respective acceptance region of fault free circuits. These regions are denoted by $A_{p}, A_{x}$, and $A_{m}$, respectively. In specification testing, a circuit is accepted if all specifications are satisfied, i.e. the measured performances $\boldsymbol{p}$ are located inside $A_{p}$. By demanding that the measurements fully characterize the state of the circuit with respect to parametric faults, modeled by the statistical parameters, specification testing is transformed into the space of measurements $\boldsymbol{m}$. A circuit is fault free if and only if the measurements $\boldsymbol{m}$ are located inside $A_{m}$. This results in the following relationship for a fault free circuit: $\boldsymbol{p} \in A_{p} \Leftrightarrow \boldsymbol{m} \in A_{m}$. In the presented approach the test design is performed based on the acceptance region $A_{m}$ in the space of measurements. Therefore, the differences between the operational environment and the testing environment are inherently considered by the proposed fault modeling. The problem arising is that, in contrast to $A_{p}$, the region $A_{m}$ is unknown. So, an approximation of this acceptance region needs to be determined.
Each given specification induces a hyperplane into the space of measurements which separates the measurement space into a region where the specification is satisfied and a region where the specification is violated $[7,12,13]$. In order to approximate the acceptance region $A_{m}$, the following proceeding is performed. Based on discrimination analysis a test criterion of the form [7, 9, 14]

$$
t(\boldsymbol{m})=\theta_{0}+\theta^{T} \boldsymbol{m} \geq 0
$$

is computed for each given specification. Thus, linear approximations of the separating hyperplanes are determined. The parameters $\theta_{0}$ and $\theta \in \mathcal{R}^{n_{m}}$ of these test criteria are computed from a training data set $[15,16,17]$. If equation (1) is satisfied the circuit is regarded as fault free with respect to the respective specification. Finally, a circuit is accepted if all individual test criteria (1) for all given specifications are satisfied.

\section{Consideration of measurement noise}

In order to provide a really applicable test design, the effects of measurement errors have to be carefully investigated and the confidence has to be studied with which the determined statistical test criteria can be applied in the presence of measurement noise [18, 19].

\subsection{Scaling}

It is assumed that the measurement errors are normally distributed with zero mean. This is true for a normal testing situation without systematic errors. The statistical distribution of the measurement $\widehat{\boldsymbol{m}}$ corresponding to an expected value $\boldsymbol{m}$ is given by $\widehat{\boldsymbol{m}} \sim \mathrm{N}\left(\boldsymbol{m}, \boldsymbol{\Sigma}_{\widehat{m}}\right)$. The contour lines of this probability density function are hyperellipsoids with center $\boldsymbol{m}$ [15]. The shape of these hyperellipsoids is determined by the covariance matrix $\Sigma_{\widehat{m}}$.

In order to obtain robust results with respect to measurement noise from discrimination analysis, a scaling of the measurements $\boldsymbol{m}$ has to be performed such that, in the space of scaled measurements, these contour lines become hyperspheres. This is because discrimination analysis can be interpreted as an optimization process that maximizes the distances of the measurements to the separating hyperplane [16]. The evaluation of distances is performed in the $l_{2}-$ norm. So, on application of the proposed scaling, the norm, which is used to calculate distances in discrimination analysis, reflects the effects of measurement noise.

The matrix that is needed to perform this scaling is determined on application of the Cholesky decomposition of $\Sigma_{\hat{m}}^{-1}$ [20]. If measurement errors are correlated, the scaled measurements cannot be interpreted in terms of physical measurements any more. Nevertheless, after discrimination analysis is performed, physically interpretable and robust test criteria are obtained in the space of unscaled measurements. 


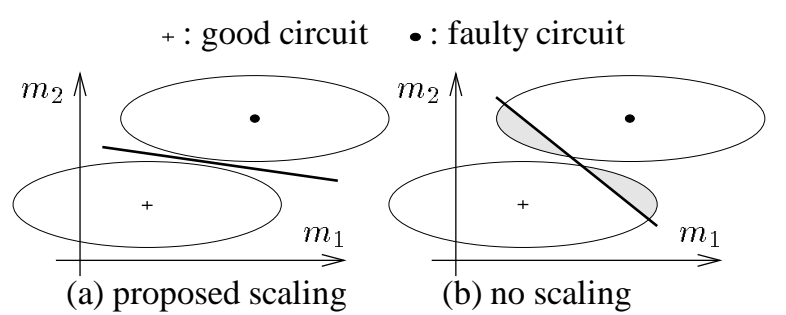

Figure 1: Comparison of the proposed scaling to the unscaled situation.

Figure 1 illustrates the advantage of the proposed scaling (a) compared to the unscaled situation (b). The test criteria that result from discrimination analysis with the respective scaling are illustrated by the solid lines. The ellipses around the two sample elements represent contour lines of the probability density function considering measurement errors. Measurements located in the shaded regions in Figure 1(b) are classified to the population that is less likely with respect to measurement errors. These shaded regions disappear if and only if the proposed scaling is applied.

\subsection{Robustness of test criteria}

Here the effects of measurement errors on the test criteria will be investigated for specification testing as well as for the presented approach. It will be shown that the specified performances are not very well suited for testing. The statistical parameters $\boldsymbol{x}$ are assumed to be multivariate normal: $\boldsymbol{x} \sim \mathrm{N}\left(\boldsymbol{x}_{0}, \boldsymbol{\Sigma}_{x}\right)$.

Specification Testing: The specified performance $p$ is assumed to be a linear function of $\boldsymbol{x}$

$$
p(\boldsymbol{x})=p_{0}+\boldsymbol{e}^{T}\left(\boldsymbol{x}-\boldsymbol{x}_{0}\right) .
$$

The vector $\boldsymbol{e}$ is the sensitivity vector of $p$ with respect to $\boldsymbol{x}$. The statistical distribution of $p$ due to fluctuations of the manufacturing process [15] is then given by: $p(\boldsymbol{x}) \sim$ $\mathrm{N}\left(p_{0}, \sigma_{p, x}^{2}=\boldsymbol{e}^{T} \boldsymbol{\Sigma}_{x} \boldsymbol{e}\right)$. With respect to the measurement error $\Delta p$, a multivariate normal distribution with zero mean is assumed: $\Delta p \sim \mathrm{N}\left(0, \sigma_{p, m}^{2}\right)$. In order to have a robust test criterion in specification testing, the variation $\sigma_{p, x}^{2}$ of the distribution of the performance $p$ due to fluctuations of the manufacturing process needs to be much bigger than the variation $\sigma_{p, m}^{2}$ of the measurement error: $\sigma_{p, x}^{2} \gg \sigma_{p, m}^{2}$.

$\sigma_{p, x}^{2}$ decreases with a small sensitivity vector $\boldsymbol{e}$. But, to design a circuit such that its performances have minimal sensitivity with respect to fluctuations of the manufacturing process is a common design goal [6]. Design objectives are in contrast to the robustness of specification testing. Thus, the specified performances are not very well suited for testing.
New Method: We assume that the measurements $\boldsymbol{m}$ depend linearly on the statistical parameters $\boldsymbol{x}$. The discrimination function $t(\boldsymbol{x})$ of test criterion (1) is then given by:

$$
\begin{aligned}
t(\boldsymbol{x})=\theta_{0}+\boldsymbol{\theta}^{T} \boldsymbol{m}(\boldsymbol{x}) & =t_{0}+\left(\boldsymbol{\theta}^{T} \boldsymbol{A}\right)\left(\boldsymbol{x}-\boldsymbol{x}_{0}\right) \\
\text { with } t_{0} & =\theta_{0}+\boldsymbol{\theta}^{T} \boldsymbol{m}\left(\boldsymbol{x}_{0}\right) ;
\end{aligned}
$$

Here, $\boldsymbol{A}$ is the sensitivity matrix of the measurements and $\boldsymbol{A}^{T} \boldsymbol{\theta}$ is the sensitivity vector of $t(\boldsymbol{x})$ with respect to $\boldsymbol{x}$. The statistical distribution of $t$ due to fluctuations of the manufacturing process [15] is then given by $t(\boldsymbol{x}) \sim$ $\mathrm{N}\left(t_{0}, \sigma_{t, x}^{2}=\boldsymbol{\theta}^{T} \boldsymbol{A} \boldsymbol{\Sigma}_{x} \boldsymbol{A}^{T} \boldsymbol{\theta}\right)$. Next, the propagation of the measurement errors $\Delta \boldsymbol{m}$ to $t$ is taken into account. As before, we assume $\Delta \boldsymbol{m} \sim \mathrm{N}\left(0, \boldsymbol{\Sigma}_{\hat{m}}\right)$. So, the error $\Delta t$ in the evaluation of $t$ [15] is given by: $\Delta t \sim \mathrm{N}\left(\mathbf{0}, \sigma_{t, m}^{2}=\right.$ $\left.\boldsymbol{\theta}^{T} \boldsymbol{\Sigma}_{\hat{m}} \boldsymbol{\theta}\right)$. Again, in order to obtain a robust test criterion in the presence of measurement noise, the following relation should be satisfied as good as possible

$$
\sigma_{t, x}^{2} \gg \sigma_{t, m}^{2} \Longleftrightarrow \theta^{T} \boldsymbol{A} \boldsymbol{\Sigma}_{x} \boldsymbol{A}^{T} \boldsymbol{\theta} \gg \boldsymbol{\theta}^{T} \boldsymbol{\Sigma}_{\hat{m}} \boldsymbol{\theta} .
$$

Please note that this relation is in good agreement to sensitivity based measurement selection approaches $[18,19]$ which aim at maximizing $\left|\boldsymbol{A}^{T} \boldsymbol{A}\right|$. On providing the degree of freedom to construct test criteria based on the most sensitive and most linearly independent measurements a robust test design is enabled. From equation (4) the following conclusion can be drawn:

The performance of the test criterion is independent on $\|\theta\|$. Increasing $\|\theta\|$ increases the sensitivity of the discrimination function $t$ with respect to the statistical parameters. But on the other hand the increased propagation of measurement errors offsets this advantage.

\subsection{Classification quality}

Evaluating the classification quality of the computed test criteria in the presence of measurement noise involves the investigation of the effect of measurement noise on the fault- and the yield coverage $[7,14,21]$. The fault coverage is the probability to detect a circuit which is faulty with respect to the considered specification. The yield coverage is the probability to accept a fault-free circuit. The worst-case absolute error in the evaluation of the discrimination function $t$ is assumed to be given by $\delta t$. We let $\delta t=3 \cdot \sigma_{t, m}$ for a confidence level of $99.7 \%$. $\delta t$ is used to compute a worst-case yield coverage $\mathrm{yc}_{\mathrm{wc}}$ and a worstcase fault coverage $\mathrm{fc}_{\mathrm{wc}}$. These conditional probabilities are given by:

$$
\begin{aligned}
\mathrm{yc}_{\mathrm{wc}} & =\mathrm{p}(t(\boldsymbol{m}) \geq+\delta t \mid \text { circuit is good }), \text { and }(5) \\
\mathrm{fc}_{\mathrm{wc}} & =\mathrm{p}(t(\boldsymbol{m})<-\delta t \mid \text { circuit is faulty })
\end{aligned}
$$




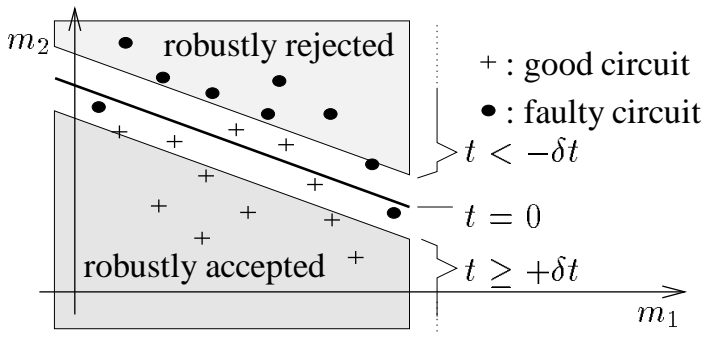

Figure 2: Classification quality in the presence of measurement noise.

As can be seen from Figure 2, by this means only sample elements that are robustly accepted or rejected in the presence of measurement noise may contribute to the worstcase yield coverage and to the worst-case fault coverage, respectively. All sample elements that are located in a $\pm \delta t$ region around the computed separating hyperplane are regarded to be falsely classified for validation purposes. Please note that this region is independent of $\|\theta\|$. This is due to the conclusion drawn from equation (4).

\section{Feature selection}

It is obvious that for the task of testing a circuit with respect to a given specification, not all measurements are of similar importance. In order to obtain a test design based on a small number of measurements, the measurements should be sorted according to the significance of information they carry for the respective testing task. This induces a possible reduction of production testing time and an improvement of the performance of discrimination analysis. The less measurements are used, the less parameters need to be estimated. Thus estimation accuracy and robustness of the test design is increased.

The task of extracting important features from the observed sample elements is called feature selection or extraction [10]. The optimal feature in linear discrimination is given by $\boldsymbol{\theta}^{T} \boldsymbol{m}$ (see [10]). This is reasonable as test criterion (1) only depends on $\boldsymbol{\theta}^{T} \boldsymbol{m}$ and the constant $\theta_{0}$. The single feature $\theta^{T} \boldsymbol{m}$ carries the same information for the considered testing task than the set of all measurements. Unfortunately, this implies no reduction of required measurements in general. The optimal feature is a linear combination of possibly all measurements.

In order to approximate the optimal feature $\boldsymbol{\theta}^{T} \boldsymbol{m}$ by a lower number of measurements, the measurements are sorted according to the absolute value of the corresponding component of $\boldsymbol{\theta}$. It is assumed that a high component value of $\theta$ indicates a high significance of the corresponding measurement. This is a heuristic based on a geometrical view of the problem. An example illustrating this, is given in Figure 3. The thick line depicts the separating

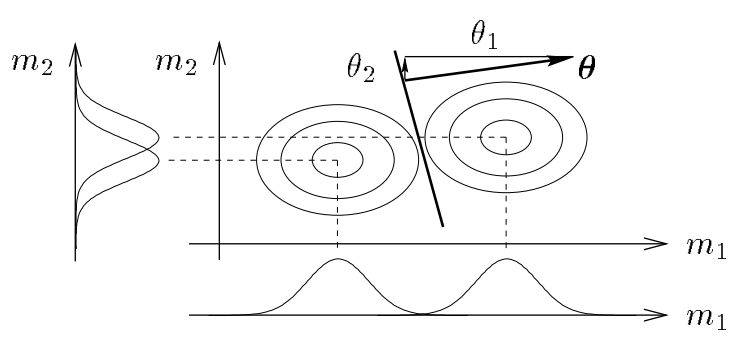

Figure 3: Illustration of the proposed heuristic for measurement selection.

hyperplane. The circles represent the contour lines of the probability density functions of the measurements for good and faulty circuits. From this figure it is obvious that measurement $m_{1}$ carries more significant information for this discrimination task than measurement $m_{2}$. When measurement $m_{1}$ is considered, the marginal probability density functions are far more separable than the ones when measurement $m_{2}$ is considered. Obviously, this corresponds to the component $\theta_{1}$ being much bigger than the component $\theta_{2}$.

Based on an increasing number of most significant measurements, test criteria for the considered specification are now computed and validated. The optimal number of required measurements is chosen interactively based on a plot, see e.g. Figure 5, showing the test quality for an increasing number of considered measurements.

\section{Sample generation}

In this section, the proceeding for the generation of training and validation samples will be addressed. These samples are required for the design and validation of the test criteria. Each set of statistical parameters $\boldsymbol{x}$ represents exactly one possibly manufactured circuit. By circuit simulations the corresponding measurements $\boldsymbol{m}$ and the corresponding performances $\boldsymbol{p}$ are evaluated. By this means sample elements in the space of measurements are obtained where for each of them the satisfaction or violation of given specifications is known.

The problem of the generation of training and validation samples is thus reduced to the generation of samples in the space of statistical parameters $\boldsymbol{x}$ of the circuit. The most straight forward approach is to generate sample elements according to the statistical distribution of the manufacturing process $\boldsymbol{x} \sim \mathrm{N}\left(\boldsymbol{x}_{0}, \boldsymbol{\Sigma}_{x}\right)$. By this means samples are generated that reproduce the manufacturing process. On application of this proceeding, a training and a validation sample are obtained that are both physically interpretable. A drawback of this proceeding is that only very few sample elements are located near the boundary of the acceptance region of a specification when it has a high individual yield.

In $[17,8]$, the authors report the importance of having 
training samples near the boundary of the acceptance region, to obtain good estimates of the parameters required for the test criterion. In order to obtain sample elements on both sides of the separating hyperplane and to retain the physically interpretability, we generate training and validation sample elements according to a normal distribution with an expected value $\boldsymbol{x}_{w}$, while keeping covariance matrix $\Sigma_{x}$. Here, $\boldsymbol{x}_{w}$ is the worst-case point [22, 12, 13] of the considered specification. The worst-case point of a specification is defined as the set of statistical parameters that just satisfies the corresponding specification and has the highest probability density to occur. So, the violation of a specification is most likely in a region around the worst-case point $\boldsymbol{x}_{w}$ of this specification. The task of worst-case point computation for individual specifications belongs to the statistical design of analog circuits.

These sample elements can be interpreted as belonging to a drifted manufacturing process. The drift is such that the considered specification is 'most critical' for testing. This means that the yield for the (linearized) specification is approximately $50 \%$. This process drift is minimal in the sense that the distance between the nominal expected value $\boldsymbol{x}_{0}$ and the expected value $\boldsymbol{x}_{w}$ of the sample distribution is minimal. This is with respect to a norm that takes into account the different variations and correlations of the statistical parameters.

Thus, for each specification a training sample $T$ and a validation sample $V$ is obtained in the space of measurements. These samples correspond to statistical parameters $\boldsymbol{x} \sim \mathrm{N}\left(\boldsymbol{x}_{w}, \boldsymbol{\Sigma}_{x}\right)$. In order to maintain a more global view on the problem, these sets are supplemented by a training sample $T_{0}$ and by a validation sample $V_{0}$ that correspond to statistical parameters $\boldsymbol{x} \sim \mathrm{N}\left(\boldsymbol{x}_{0}, \boldsymbol{\Sigma}_{x}\right)$.

For the computation of the test criterion for a given specification, the union of the global training sample $T_{0}$ and the respective training sample $T$ is used. For validation of a single test criterion, the respective validation sample $V$ is considered. Validation of the overall test design is done by the global validation sample $V_{0}$.

\section{Consideration of catastrophic faults}

In this section, a very efficient method for the handling of catastrophic faults will be presented. A circuit is regarded as faulty not only if any given specification is not satisfied but also if the set of statistical parameters that correspond to the circuit under test indicates a very large deviation from the nominal design. Such a circuit should not be accepted, because a parameter deviation above a certain limit indicates a production fault. In the following, the proposed proceeding for the detection of these faults will be illustrated.

\subsection{Bounding the acceptance region}

In many cases, the acceptance region $A_{x}$ in the space of statistical parameters is not bounded. This means that a circuit with parameters very far away from the nominal design may satisfy all given specifications. By additionally introducing box constraints for the measurements, the acceptance region $A_{m}$ in the measurement space can easily be bounded: $\boldsymbol{m}^{L} \leq \boldsymbol{m} \leq \boldsymbol{m}^{U}$. As the measurements completely characterize the state of the circuit with respect to the statistical parameters, this induces a bounded acceptance region of the test in the space of statistical parameters, too. The boundary values are rapidly obtained by using all training sample elements $\boldsymbol{m}$ generated for discrimination analysis: $\left(1 \leq i \leq n_{m}\right)$

$$
m_{i}^{L}=\min _{\boldsymbol{m} \in A_{m}} m_{i}-\varepsilon_{i}, \quad m_{i}^{U}=\max _{\boldsymbol{m} \in A_{m}} m_{i}+\varepsilon_{i},
$$

where $\varepsilon_{i}$ are positive constants chosen by the test engineer. Now, a circuit is accepted if all test criteria and the box constraints are satisfied. Please note that this extension of the definition of a faulty circuit is based on similar considerations as in $[8,9,23]$. In the approach proposed in this paper, these rather crude test criteria take effect only when no given specification induces a reasonable limit for permissible parameter deviations.

The simulated results show that the application of these box constraints improves the fault coverage with respect to catastrophic faults, while test efficiency for parametric faults is not reduced.

\subsection{Test set compaction}

In order to be able to handle catastrophic faults, a fault list of these faults needs to be generated. Here, the four most probable device faults are considered for each transistor. Shorts between gate and drain, and gate and source, an open source contact, and an open drain contact [24] are modeled. Of course, methods for inductive fault analysis could also be applied for construction of the fault list.

For this fault list, an analog fault simulation is performed, i.e. the corresponding measurements are determined. In order to save computation time, only the nominal circuit design is considered here.

With the results of this fault simulation, a test set compaction [25] is performed, i.e. all measurements that are not necessarily required for the test design are removed. For each modeled fault, all tests which detect that fault are determined. A test is either a test criterion that was designed for testing a given specification, or it consists of two box constraints concerning the lower and upper boundary values for one measurement $\left(m_{i}^{L} \leq m_{i} \leq m_{i}^{U}\right)$. A test table is then formed containing a row for each test, a column for each fault and the entry in row $\mu$ and column $\nu$ is 1 if and only if the test $\mu$ detects the fault $\nu$. In order to find a 


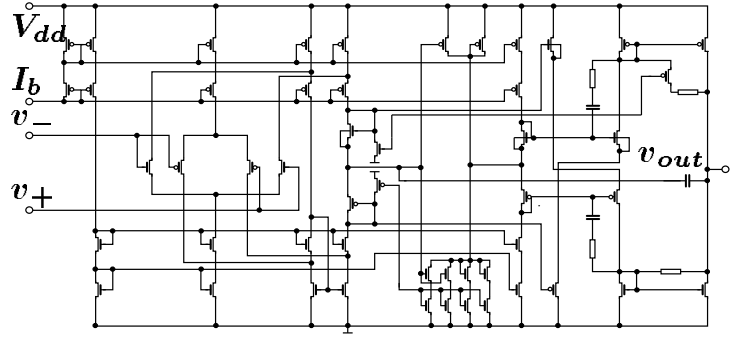

Figure 4: Circuit to be tested.

\begin{tabular}{|c|c|c|c|c|}
\hline Gain & GBW & $\mathrm{SR}_{-}$ & $\mathrm{SR}_{+}$ & $\Delta V_{\text {out }}$ \\
\hline$>69 \mathrm{~dB}$ & $>2.5 \mathrm{MHz}$ & $>4.0 \mathrm{~V} / \mu \mathrm{s}$ & $>4.0 \mathrm{~V} / \mu \mathrm{s}$ & $>3.8 \mathrm{~V}$ \\
\hline
\end{tabular}

Table 1: Specifications given for the circuit.

minimal set of measurements to detect all detectable faults, the following proceeding is performed: First, all faults are removed that are detected by the designed test criteria and by the box constraints that concern already used measurements. The problem of finding a minimal number of additional tests, such that all faults that can be detected by the set of all measurements, are detected by the set of selected tests, too, can be formulated as a covering problem [25]. Here a well known heuristic is used: The test that detects the highest number of faults is added to the list of measurements that really have to be measured in the actual testing phase. Then, these faults are removed from the fault list. This is repeated until the fault list contains only faults that are not detectable by any remaining test. On termination of this simple but very efficient heuristic, a nearly minimal set of additional measurements has been determined that is required for testing the circuit with respect to catastrophic faults.

Modeled catastrophic faults that cannot be detected by the considered measurements are not proven to be redundant. For these faults any known method for the detection of catastrophic faults may be applied. The efficiency of these methods generally depends on the number of catastrophic faults that need to be considered. Thus, by first performing the test design with respect to parametric faults and exploiting the results as efficiently as possible with respect to catastrophic faults, leads to an improved starting position for methods dealing with the detection of catastrophic faults.

\section{Experimental results}

For the complex CMOS operational amplifier shown in Figure 4, the test design was performed. The specifications in Table 1 need to be guaranteed to the customer. For testing, the operational amplifier is configured as a voltage follower. All accessible quantities are considered to be mea-

\begin{tabular}{|c|c|cc|cc|}
\hline$U$ & $1.5 \mathrm{~V}$ & \multicolumn{2}{|c|}{$2.5 \mathrm{~V}$} & \multicolumn{2}{c|}{$3.5 \mathrm{~V}$} \\
\hline$\omega$ & DC & DC & $670 \mathrm{kHz}$ & DC & $100 \mathrm{kHz}$ \\
& $1.60 \mathrm{MHz}$ & $1.09 \mathrm{MHz}$ & $1.60 \mathrm{MHz}$ & $670 \mathrm{kHz}$ & $1.60 \mathrm{MHz}$ \\
\hline
\end{tabular}

Table 2: Considered input stimuli.

surable, i.e. the input current, the currents over the power supply voltages, the voltage at the inverting input, and the output voltage. Parametric faults of the amplifier are modeled by multiple deviations of twelve statistical model parameters. They represent perturbations in the transistor geometries $\Delta W$ and $\Delta L$, in the oxide thickness $t_{o x}$, the mobilities $\mu_{0, p / n}$, the threshold voltages $V_{T 0, p / n}$, the bulk threshold parameters $\gamma_{p / n}$, the junction capacitances $c_{j, p / n}$ (for $\mathrm{p}$ - and n-type transistors) and the bias current. The considered DC and AC input stimuli are listed in Table 2. These measurements were selected by a sensitivity based measurement selection procedure [18, 19]. The number of measurements in a DC or an AC test is five or ten, respectively. So, $n_{m}=85$ measurements of the circuit are available. The measurement errors are assumed to be not correlated and worst-case measurement errors of $0.1 \%$ of the nominal values are assumed. 1000 global training sample elements $\left(T_{0}\right)$ and 4000 global validation sample elements $\left(V_{0}\right)$ are generated. For each of the five specifications, the individual training samples $T$ consist of 1000 sample elements and the individual validation samples $V$ consist of 500 sample elements. The overall number of simulations was 12,500 .

\subsection{Design of individual test criteria}

The test criteria, equation (1), are computed for each of the five specifications considering all $n_{m}$ measurements. By application of the proposed heuristic for feature selection an ordering of measurements with respect to their significance for the respective testing task is obtained. Next, the test criteria are computed once again considering only the $n$ most significant measurements $\left(n=1, \ldots, n_{m}\right)$. Figure 5 shows the quadratic mean of the fault- and the yield coverage for an increasing number of considered measurements for the test design for specification $\mathrm{SR}_{-}$. In a first phase, the quality of the test increases significantly with the number of considered measurements. After this phase, the quality of the test does not improve any more. This is true for the other specifications as well. For testing the specification $\mathrm{SR}_{-} 16$ measurements are sufficient.

The yield- and fault coverages for all five test criteria are given in Table 3. Validation is done with the respective validation sample $V$. Thus, the test design is validated with respect to the most critical process drift for the individual specification as discussed in section 5. This table shows that the consideration of measurement noise leads to a slight decrease in the yield- as well as in the fault cover- 


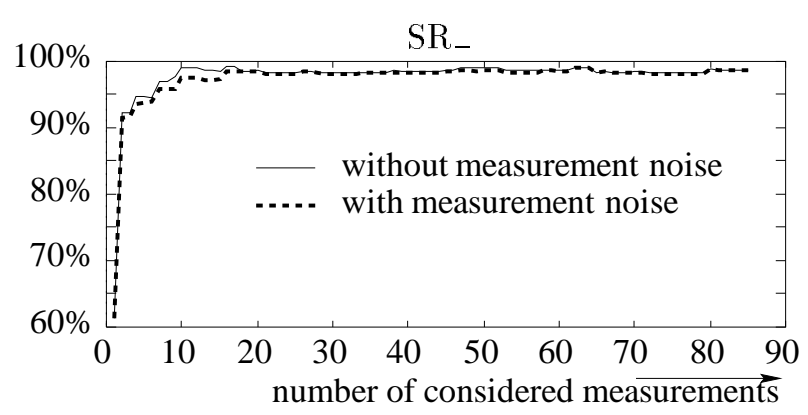

Figure 5: Quadratic mean of fault-and yield coverage for the test design for specification $\mathrm{SR}_{-}$.

\begin{tabular}{|l||c||c|r||r|r||r|}
\hline Spec. & yield & $\mathrm{yc}$ & \multicolumn{1}{c||}{$\mathrm{yc}_{\mathrm{wc}}$} & \multicolumn{1}{c|}{$\mathrm{fc}$} & \multicolumn{1}{|c|}{$\mathrm{fc}_{\mathrm{wc}}$} & $n^{\star}$ \\
\hline \hline Gain & $53.1 \%$ & $97.2 \%$ & $96.4 \%$ & $100.0 \%$ & $100.0 \%$ & 28 \\
GBW & $48.0 \%$ & $91.0 \%$ & $88.2 \%$ & $100.0 \%$ & $100.0 \%$ & 2 \\
$\mathrm{SR}_{-}$ & $48.3 \%$ & $95.7 \%$ & $95.3 \%$ & $99.6 \%$ & $99.2 \%$ & 16 \\
$\mathrm{SR}_{+}$ & $98.7 \%$ & $98.5 \%$ & $98.3 \%$ & $100.0 \%$ & $100.0 \%$ & 12 \\
$\Delta V_{\text {out }}$ & $53.3 \%$ & $98.3 \%$ & $98.3 \%$ & $99.5 \%$ & $99.5 \%$ & 8 \\
\hline
\end{tabular}

Table 3: Yield- and fault coverages for the individual test criteria. The subscript wc denotes the consideration of measurement noise. The last column shows the number of used measurements.

age. Nevertheless, if another scaling than the one presented in section 3.1 is performed before discrimination analysis is carried out, a strong degradation of the quality of the test can be observed when measurement noise is taken into account. If, e.g., a scaling is performed such that all measurements are located inside the unity cube, both, the worst-case yield- and fault coverages for the testing of, e.g., specification Gain, drop to $0 \%$. Such test criteria are absolutely meaningless. This emphasizes the necessity of systematically considering measurement noise in all stages of the test design.

A more detailed diagnosis of misclassified circuits for specification $\mathrm{SR}_{-}$is given in Figure 6. It can be easily seen that the performance values of misclassified circuits are very close to the specified boundary value of $4.0 \mathrm{~V} / \mu \mathrm{s}$ (see Table 1), represented by the thick dashed line. This statement is true for the other specifications as well.

\subsection{Validation of the total test criterion}

The results for the conjunction of all individual test criteria are shown in Table 4. The difference in the results between Table 3 and Table 4 is not only due to the fact that Table 4 gives the results for the conjunction of all five test criteria but also due to the different samples that are used for validation. Considering Tables 3 and 4 together, it can be seen that the proposed method performs very good with respect to the detection of parametric faults. All 4000 vali-

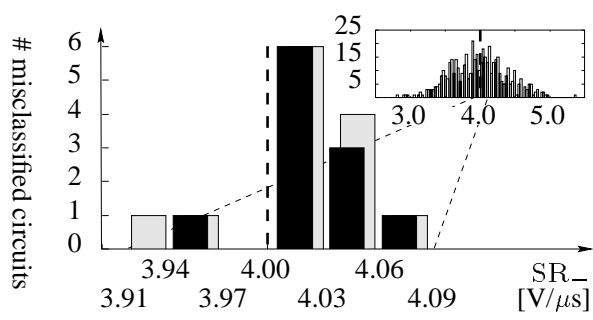

Figure 6: Histogram of performance values of misclassified circuits for specification $\mathrm{SR}_{-}$. Dark shaded bars show results without and light shaded bars show results with consideration of measurement noise. Top right histogram shows the total distribution of the performance $\mathrm{SR}_{-}$for the validation sample $V$.

\begin{tabular}{|c||c||c|c||c|c||c|}
\hline Spec. & yield & $\mathrm{yc}$ & $\mathrm{yc}_{\mathrm{wc}}$ & $\mathrm{fc}$ & $\mathrm{fc}_{\mathrm{wc}}$ & $n_{\text {tot }}^{\star}$ \\
\hline \hline tot. circ. & $99.9 \%$ & $100.0 \%$ & $100.0 \%$ & $100.0 \%$ & $100.0 \%$ & 35 \\
\hline
\end{tabular}

Table 4: Yield- and fault coverages for the total test criterion. The subscript wc denotes the consideration of measurement noise. Validation is performed with validation sample $V_{0}$. The last column gives the total number of used measurements.

dation sample elements that characterize the nominal manufacturing process are correctly tested. From Table 4 it can be seen that the total number of measurements required for the test design with respect to parametric faults is only 35 . This is much less than the sum of measurements required for the testing of the five individual specifications. This is due to the fact that sometimes the same measurements are found to be significant for the individual testing tasks.

\subsection{Consideration of catastrophic faults}

The modeling of catastrophic faults described in section 6.2 results in a fault list with 164 faults for this circuit when the single fault assumption is applied and designed shorts as e.g. in current mirrors are removed. With this fault list, analog fault simulation and test set compaction is performed. The results for specification testing and for the proposed method, without and with box constraints for measurements that are already required for the detection of parametric faults are shown in Table 5. The last row of this table shows the results of the heuristic for the fault covering problem. Only three additional measurements of the circuit need to be performed to detect all 156 detectable catastrophic faults. Eight faults cannot be detected by any of the considered tests. With respect to specification testing a circuit is regarded as fault free if all specifications of Table 1 are satisfied. Please note that no additional DC measurements are considered here.

It can be seen that the proposed method especially 


\begin{tabular}{|l||c|c|}
\hline 164 faults considered & \# tested & $n_{\text {tot }}^{\star}$ \\
\hline \hline specification testing & 94 & - \\
\hline \hline test criteria & 101 & 35 \\
\hline $\begin{array}{l}\text { test criteria \& box constraints } \\
\text { for used measurements }\end{array}$ & 121 & 35 \\
\hline $\begin{array}{l}\text { test criteria \& box constraints } \\
\text { for maximal coverage }\end{array}$ & 156 & 38 \\
\hline
\end{tabular}

Table 5: Results for catastrophic faults.

with additionally considered box constraints features a very large number of detected modeled catastrophic faults, while specification testing lags behind.

\section{Conclusion}

In this paper, a new and very efficient method for the design of robust test criteria is presented. For each given specification, a test criterion is computed based on discrimination analysis. These test criteria are very robust with respect to measurement noise. This is due to the following considerations: First, measurement noise is systematically considered in all stages of the test design. Second, by application of methods for feature selection the computed test criteria are based on a minimal number of measurements that are most significant for the testing of the respective specification. Third, on applying a novel sampling strategy both a more global as well as a local view on the problem is provided. By this means samples are generated that are especially meaningful for the respective testing task. By applying these test criteria, the satisfaction or violation of the original circuit specifications can be reliably inferred from the measurements of the circuit under test. Moreover, on application of digital testing methods the test design is extended to cover catastrophic faults, at low additional test cost. The simulated results demonstrate the very good performance of the proposed method with respect to parametric as well as to catastrophic faults.

\section{References}

[1] P. Fieler and N. Loverro. Defects Tail Off with SixSigma Manufacturing. IEEE Circuits and Devices Magazine, 7(5):18-20,48, September 1991.

[2] M. Soma. Challenges in analog and mixed-signal fault models. IEEE Circuits and Devices Magazine, pages 1619, January 1996.

[3] S. Millman. Improving quality: Yield versus test coverage. JETTA, 5:253-261, 1994.

[4] L. Milor and A.L. Sangiovanni-Vincentelli. Minimizing Production Test Time to Detect Faults in Analog Circuits. IEEE Trans. CAD, 13:796-813, 1994.

[5] J.B. Brockman and S.W. Director. Predictive Subset Testing: Optimizing IC Parametric Performance for Quality, Cost and Yield. IEEE Trans. Semiconductor Manufacturing, 2:104-113, 1989.
[6] S.W. Director and W. Maly, editors. Statistical Approach to VLSI, volume 8. Elsevier Science B.V., Amsterdam, Advances in CAD for VLSI edition, 1994.

[7] W.M. Lindermeir, H.E. Graeb, and K.J. Antreich. Design Based Analog Testing by Characteristic Observation Inference. In Proc. IEEE ICCAD, pages 620-626, November 1995.

[8] A. Wu and J. Meador. Measurement Selection for Parametric IC Fault Diagnosis. JETTA, 5:9-18, 1994.

[9] B.R. Epstein, M. Czigler, and S.R. Miller. Fault Detection and Classification in Linear Integrated Circuits: An Application of Discrimination Analysis and Hypothesis Testing. IEEE Trans. CAD, 12:102-113, 1993.

[10] K. Fukunaga. Introduction to Statistical Pattern Recognition. Computer Science and Scientific Computing. Academic Press, San Diego, second edition, 1990.

[11] J.L. Huertas. Test and Design for Testability of Analog and Mixed-Signal Integrated Circuits: Theoretical Basis and Pragmatical Approaches. Proc. ECCTD, pages 77-156, 1993.

[12] A. Dharchoudhury and S. M. Kang. Worst-Case Analysis and Optimization of VLSI Circuit Performances. IEEE Trans. CAD, 14(4):481-492, April 1995.

[13] K.J. Antreich, H.E. Graeb, and C.U. Wieser. Circuit Analysis and Optimization Driven by Worst-Case Distances. IEEE Trans. CAD, 13:57-71, 1994.

[14] Z. Wang, G. Gielen, and W. Sansen. Testing of Analog Circuits Based on Power-Supply Current Monitoring and Discrimination Analysis. In IEEE Asian Test Symposium, pages 126-131, November 1994.

[15] R.A. Johnson and D.W. Wichern. Applied Multivariate Statistical Analysis. Prentice Hall, Englewood Cliffs, New Jersey, third edition, 1992.

[16] N.E. Day and D.F. Kerridge. A General Maximum Likelihood Discriminant. Biometrics, 23:313-323, 1967.

[17] J.A. Anderson. Separate Sample Logistic Discrimination. Biometrika, pages 19-35, 1972.

[18] G.J. Hemink, B.W. Meijer, and H.G. Kerkhoff. Testability Analysis of Analog Systems. IEEE Trans. CAD, 9:573-583, 1990.

[19] G.N. Stenbakken and T.M. Sounders. Test-Point Selection and Testability Measures via QR Factorization of Linear Models. IEEE Transactions on Instrumentation and Measurement, 36:406-410, June 1987.

[20] G.H. Golub and C.F. van Loan. Matrix Computations. 2nd Edition, The Johns Hopkins University Press, 1989.

[21] G. Gielen, Z. Wang, and W. Sansen. Fault Detection and Input Stimulus Determination for the Testing of Analog Integrated Circuits Based on Power-Supply Current Monitoring. Proc. IEEE ICCAD, pages 495-498, 1994.

[22] K. Krishna and S.W. Director. The Linearized Performance Penalty (LPP) Method for Optimization of Parametric Yield and Its Reliability. IEEE Trans. CAD, 14(12):1557-1568, December 1995.

[23] N.B. Hamida and B. Kaminska. Multiple Fault Analog Circuit Testing by Sensitivity Analysis. Analog Integrated Circuits and Signal Processing, 4:231-243, 1993.

[24] L. Milor and V. Visvanathan. Detection of Catastrophic Faults in Analog Integrated Circuits. IEEE Trans. CAD, 8:114-130, 1989.

[25] Miron Abramovici, Melvin A. Breuer, and Arthur D. Friedman. Digital Systems Testing and Testable Design. Computer Science Press, New York, 1990. 